\title{
WIND TUNNEL TESTS AND WIND-INDUCED VIBRATION ANALYSIS OF SPHERICAL DOMES
}

\author{
Zhi-hong Zhang* and Yukio Tamura \\ Wind Engineering Research Center, Tokyo Polytechnic University, 1583 Iiyama Atsugi Kanagawa 243-0297 Japan \\ *(Corresponding author: E-mail: zhangzh@arch.t-kougei.ac.jp; Tel.: +81-46-242-9928; Fax: +81-46-242-9928)
}

\begin{abstract}
A series of wind pressure measurements on the surfaces of spherical domes are carried out in an atmospheric boundary-layer wind tunnel. The wind pressure distributions on the surfaces of spherical domes including mean and standard deviation are presented. Effects on wind pressure distribution due to wall-height-to-span ratio and rise-to-span ratio, terrain type and Reynolds number are discussed. This study focuses on wind-induced vibration analysis. The Proper Orthogonal Decomposition (POD) technique is adopted to reconstruct the wind pressure field for reticulated spherical domes with different mesh size and shape, and compared with those obtained from a wind-tunnel test model. A new treatment of non-uniformly distributed taps is proposed. Different treatments lead to different optimal problems with different physical meanings. For mode superposition analysis of wind-induced vibration analysis, a new matrix, designated by the authors as a mode-load-correlation matrix, is proposed to determine the special mode that contributes most to the wind effects. This mode contributes most to the background response and significantly to the resonant part. The physical meaning of this matrix is the spatial distribution of structural response. The advantage is that it only takes into account the known variables in the motion equation, without any quasi-static or dynamic assumption. Finally, the application of this matrix to background response is presented.
\end{abstract}

Keywords: Wind tunnel test; wind pressure; wind-induced vibration; spherical dome; reticulated shell; high modes effect

\section{LIST OF SYMBOLS}

$\begin{array}{ll}Z_{G} & \text { thickness of atomospheric boundary layer } \\ \alpha & \text { power law of terrain types } \\ f & \text { rise of spherical dome } \\ h & \text { height of surrounding wall of rigid models } \\ D & \text { span of spherical dome } \\ C_{\bar{p}} & \text { mean wind pressure coefficient } \\ \bar{p} & \text { mean wind pressure } \\ q_{H} & \text { reference wind pressure } \\ C_{\sigma_{p}} & \text { wind pressure standard deviation coefficient } \\ \sigma_{p} & \text { wind pressure standard deviation } \\ \phi(x, y) & \text { proper function } \\ \Delta S_{i} & \text { tributary area of each pressure tap } \\ {\left[R_{p}\right]} & \text { spatial correlation matrix of wind pressure } \\ \left\{\phi^{\prime}\right\} & \text { POD modes weighted by tributary area } \\ \lambda & \text { POD mode eigenvalues } \\ a_{j}(t) & \text { principal coordinates of POD analysis } \\ W\left(x_{i}\right) & \text { weighted function due to tributary area } \\ {[M]} & \text { mass matrix } \\ {[C]} & \text { damping matrix } \\ {[K]} & \text { stiffness matrix } \\ \{Y(t)\} & \text { displacement response of structure } \\ {\left[\phi^{\prime}\right]} & \text { POD modes matrix }\end{array}$




$\begin{array}{ll}\{a(t)\} & \text { matrix of principle coordinates } \\ \{P(t)\} & \text { external fluctuating load } \\ {[\varphi]} & \text { modal matrix that is independent of time } \\ \{q(t)\} & \text { modal coordinates } \\ \{q(t)\}_{B} & \text { background response } \\ \{q(t)\}_{R} & \text { resonant response } \\ {[M L C]} & \text { mode-load correlation matrix }\end{array}$

\section{INTRODUCTION}

A series of wind pressure measurements have been carried out in Wind Engineering Research Center of Tokyo Polytechnic University in order to detect the wind effects on large-span reticulated spherical shells. The author considers that, for a long time for large-span reticulated shells, there has been no consensus on high mode effects in mode superposition transient analysis. The motivation for this study was to determine this high-order "fundamental" mode.

Taniguchi et al. [1] measured the time-averaged pressure distribution over the surface of a hemisphere immersed in a fully developed turbulent boundary layer developing along a smooth, plane wall. The drag coefficient and lift coefficients were also studied. Toy et al. [2,3] investigated a hemispherical dome and a semi-cylindrical dome immersed in a boundary-layer wind tunnel. Newman et al. [4] measured the pressure distribution on three domes with different rise-to-span ratios ( $f / D=0.5,0.37,0.25)$. Cook [5] gave out some analytical formulas for wind pressure coefficient distribution of a semi-cylinder and a hemisphere in smooth uniform flow. Taylor [6] did a comprehensive study of the aerodynamic pressure distribution on hemispherical domes in boundary layer flows, including the determination of the mean, minimum, maximum and area-average pressure coefficients. The critical area and critical pressures for design were defined. Hongo [7] carried out a series of wind tunnel experiments on mean and fluctuating wind pressures with different rise-to-span ratios and different wall-height-to-span ratios.

For wind pressure field reconstruction on indirectly measured points on a model surface, Uematsu and Yamada [8] applied POD to investigate the structure of the pressure field on spherical domes and to generate wind loads at the interior nodes of the network by superimposing the first eight eigenfunctions of pressure obtained from POD analysis. They considered that mismatch between high-frequency pressure fluctuations of experiment and simulation may not significantly affect the dome's general response. This is because the spatial correlation of these pressure fluctuations is rather low and their frequencies are much higher than the dome's natural frequencies. Y. Uematsu et al. [9] carried out some basic investigations on wind-induced dynamic behavior and the resultant load estimation of a single-layer lattice dome with a large span. POD is used to generate the wind pressure time history at nodes measured indirectly. Carassale et al. [10] studied POD both in the time domain (Covariance Proper Transformation) and in the frequency domain (Spectral Proper Transformation).

For non-uniformly distributed taps of wind pressure measurement Jeong et al. [11] discussed POD applied to wind pressure specified at non-uniformly distributed pressure taps. The author considers that the first weighted method of the correlation/covariance matrix proposed by them leads to a non-symmetric covariance matrix, which is not good for the computational solution of eigenvalue problems. The second weighted method that needs both a weighted correlation/covariance matrix and a weighted POD mode leads to a different optimal mechanical 
problem. Thus, we re-derive the whole process of POD in Section 4.1. It is clear that there is a simple way to take account of the non-uniformly distributed taps' effect [12].

For wind-induced vibration analysis of single-layer reticulated spherical shells, Masuda et al. [13] and Nakayama et al. [14] proposed an efficient selection of dominant modes from the viewpoint of contribution to the expected fluctuating strain energy of the system. It was pointed out there existed a specific mode "X-mode", which was indispensable in estimating wind response, even though its natural frequency or modal order may be very high. A method for predicting the "quasi-X-mode" was also proposed. Ni et al. [15] studied the wind-induced response of a dome-like roof using the load-dependent Ritz vector method proposed by Wilson [16,17]. He and Dong [18] proposed a new frequency domain method for wind response analysis of spatial lattice structures with mode superposition. This is termed a mode compensation method. The authors of this paper [19] presented a new way for mode truncation by checking the relationship between the eigenpairs from Proper Orthogonal Decomposition analysis and Finite Element Method modes. This paper gives out the details of our idea.

Recently Li and Tamura [20,21] published their work on wind effects on a single-layer reticulated spherical dome. An equivalent static wind loading estimation method from the viewpoint of stability was also proposed.

Wind pressure distribution affected by wall-height-to-span ratio, rise-to-span ratio and Reynolds number is presented. A new treatment of POD analysis for reconstruction of the wind pressure field on the surface of reticulated spherical shells is discussed. The second objective of this paper is to investigate wind effects on large-span reticulated spherical shells. A new simple way to find out the special mode that contributes most to the wind effects is put forward. This mode contributes most to background response and significantly to the resonant part. A new matrix (mode-load-correlation matrix) is proposed. It is concluded that this matrix can reveal the spatial distribution of fluctuating structural response including the background response and the resonant response. Some suggestions are also made for further research.

\section{WIND PRESSURE MEASUREMENT EXPERIMENTS OF SPHERICAL DOMES}

\subsection{Wind Fields Simulation}

Four parameters are required to simulate the natural atmospheric boundary layer in the wind tunnel: mean wind speed profile, turbulence intensity profile, turbulence scale and power spectrum. Wind pressure measurements of spherical domes were carried out in the wind tunnel of the Wind Engineering Research Center of Tokyo Polytechnic University. This wind tunnel has a work section $15 \mathrm{~m}$ long, $2.2 \mathrm{~m}$ wide and $1.8 \mathrm{~m}$ high. A thickness $Z_{G}=100 \mathrm{~cm}$ of two boundary layers with terrain types with power laws $\alpha=0.195,0.346$ is generated using a set of spires and a number of roughness blocks on the wind-tunnel floor.

\subsection{Spherical Dome Models and Wind Pressure Measurements}

The geometry of the wind pressure measurement model is shown in Figure 1. 12 models of two terrain types (III, V) with rise-to-span ratios $f / D=1 / 3,1 / 5$ (Model 1,2) and wall-height-to-span ratios $h / D=1,2 / 3,1 / 2,1 / 3,1 / 6$ and 0 (Case 1 6) are measured. The wind pressure distribution of both the spherical dome and the wall surface are measured synchronously but separately. 
Shells with rise-to-span ratios smaller than $1 / 5$ are regarded as shallow shells. In this study, the length scale of the models is assumed to be 1/400. Thus, the dynamic responses of prototype shells with $120 \mathrm{~m}$ spans are analyzed later. The model surfaces were smooth. Each model had 91 pressure taps nearly uniformly distributed on the dome surface (K6-6, Figures 2 and 3). The maximum number of pressure taps on the wall was 108 . The frequency of the low pass filter was set to $300 \mathrm{~Hz}$. The sampling frequency of synchronous pressure measurement was $1000 \mathrm{~Hz}$. Tube correction is carried out by using a flat panel with 32 taps and a white noise generation machine. The test wind velocities were set to $10 \mathrm{~m} / \mathrm{s}, 8 \mathrm{~m} / \mathrm{s}$ and $6 \mathrm{~m} / \mathrm{s}$ at the top of model (Case 1) corresponding to a full-scale wind speed of $60 \mathrm{~m} / \mathrm{s}$. The sampling interval was $15 \mathrm{~s} / \mathrm{time}$, and each case was tested 10 times. The time interval was $0.04 \mathrm{~s}$ in full scale. The blockage effect in all of the measurements is considered small enough to be ignored.

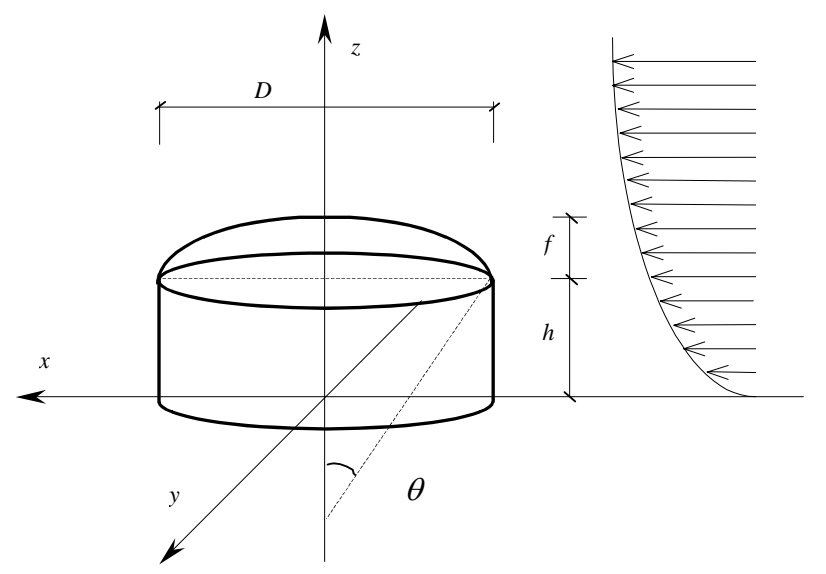

Figure 1. Dome model geometry

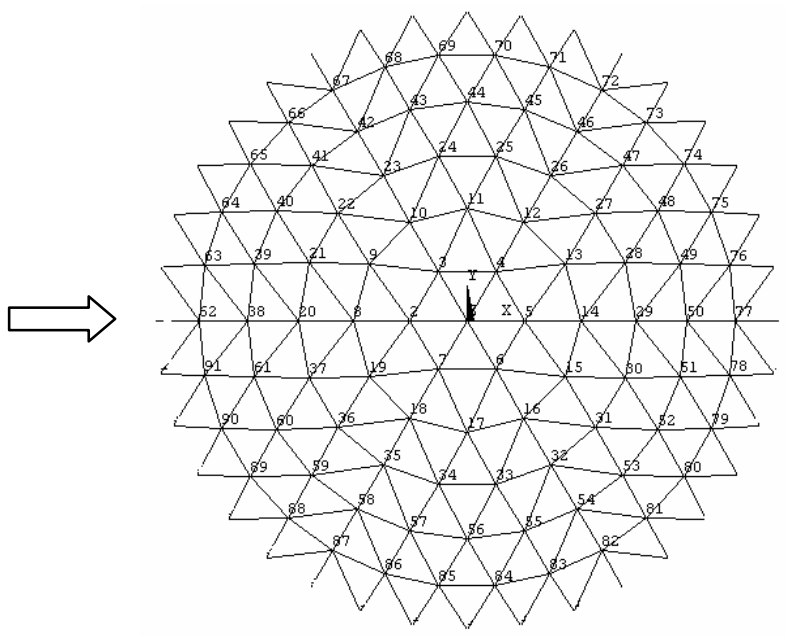

Figure 2. Numbering of pressure taps (K6-6)
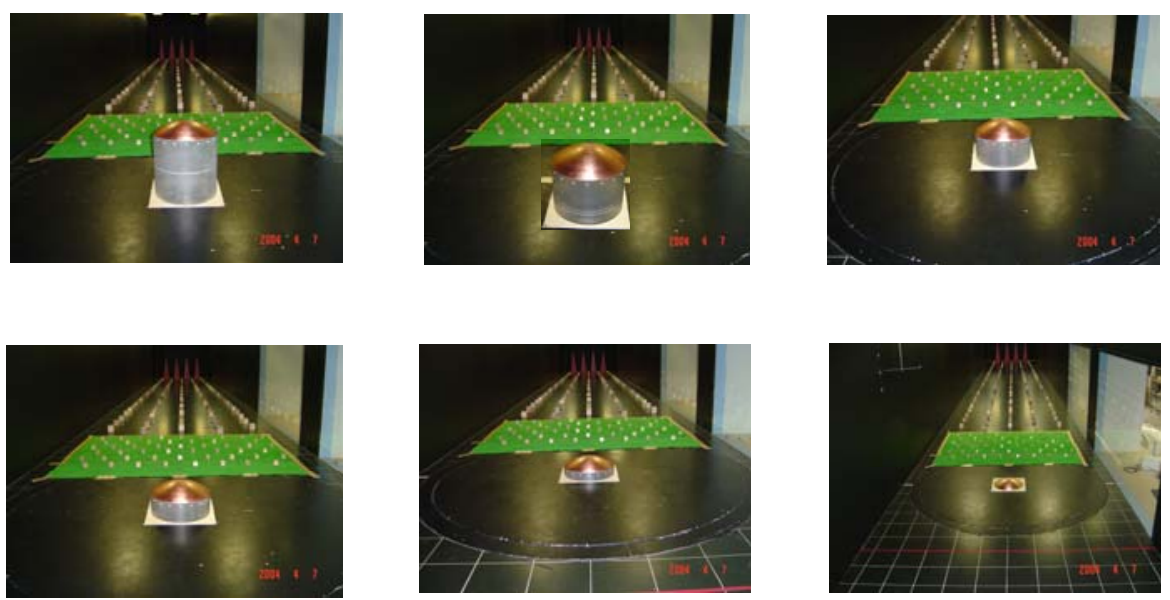

Figure 3. Test model (Case 1 6, Terrain type Ш, Taps distribution K6-6)

\section{EXPERIMENTAL RESULTS}

This section gives out experimental results from wind pressure measurements in details.

\subsection{Definition of Pressure Coefficients}

The wind pressure coefficients are defined in terms of the pressure at the top point of the roof model. 
Mean

Standard Deviation

$$
C_{\bar{p}}=\bar{p} / q_{H}
$$$$
C_{\sigma_{p}}=\sigma_{p} / q_{H}
$$

Where $\bar{p}$ is the mean pressure, $q_{H}$ is the reference wind velocity pressure at the reference height H (top of model).

\subsection{Effect of Different Wall-height-to-Span Ratios}

Figures 4 and 5 show the wind pressure coefficients $C_{\bar{p}}$ and $C_{\sigma_{p}}$, respectively, of model 1 in terrain type $\amalg$ with six different wall-height-to-span ratios. With decrease of wall height, the curves move integrally upward, since the turbulence intensity increases while the mean wind velocity decreases.

\subsection{Effect of Different Terrain Types}

Figure 6 shows the mean wind pressure coefficient distribution of model 1 in terrain types $\amalg$ and V with reference wind velocity $10 \mathrm{~m} / \mathrm{s}$. This shows that different terrain types give rise to different wind pressure distributions.

\subsection{Reynolds Number Effect}

Figures 7 and 8 show the mean and standard deviation coefficient distribution of model 1 in Terrain type $\amalg$ under three different reference velocities at the top of the roof. The Reynolds number is between $1.0 \times 105$ and $3.0 \times 105$ or so (subcritical). It is found that different reference wind velocities could affect the mean and the standard deviation wind pressure coefficient. This is consistent with that of Taylor [6] and Hongo [9]. It is proposed to carefully choose appropriate reference wind velocities for ABLWT tests on streamlined structures. In the practical situation (full scale dome) the Reynolds number is about 108 109 (Transcritical). It is nearly impossible to satisfy the Reynolds number simulation requirement in ABLWT tests. It is mentioned by Taylor [6] that for the turbulence intensity of natural wind $(>0.15 \%)$ the wind pressure is considered relatively insensitive when the Reynolds number is above $1.7 \times 105$. The main objective of this paper is not to detect the Reynolds number effect, but this is very important in practical design. A similar flow pattern corresponding to a high Reynolds number is usually generated by increasing the surface roughness or using tripping wires [22].

\section{WIND-INDUCED VIBRATION ANALYSIS}

There are three steps in analyzing the wind-induced vibration of reticulated spherical domes. First, convert the wind pressure time history of the reduced-scale model to that of the full-scale model. This will change the amplitude of the wind pressure and sampling frequency. Second, reconstruct the wind field, because there will usually be some nodes (practical lattice shell) that have not been measured. The efficiency and validation of POD in reconstructing the wind field are investigated using half the taps on the reduced-scale model to reconstruct the wind pressure time history of all of the taps [12]. Third is transient analysis of wind-induced response. It is not easy to determine the special predominant mode when the superposition method is adopted. A new way to find this mode is proposed in Sec. 4.2 . 


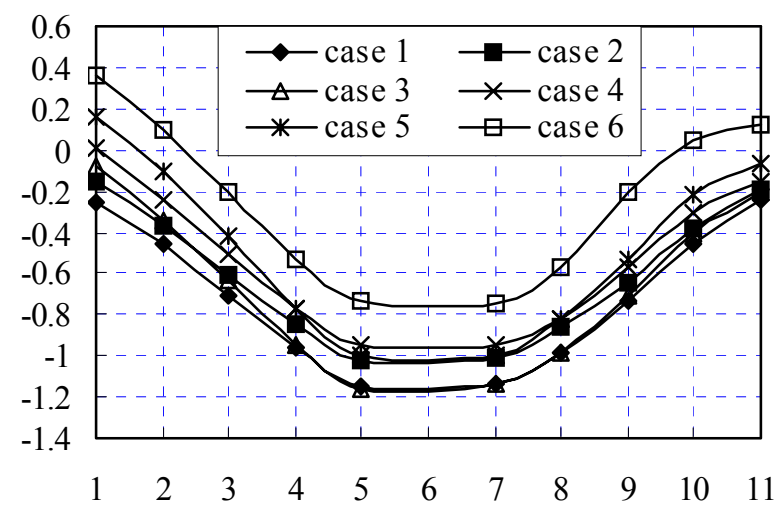

Figure 4. Mean value of Model 1,

Terrain type Ш

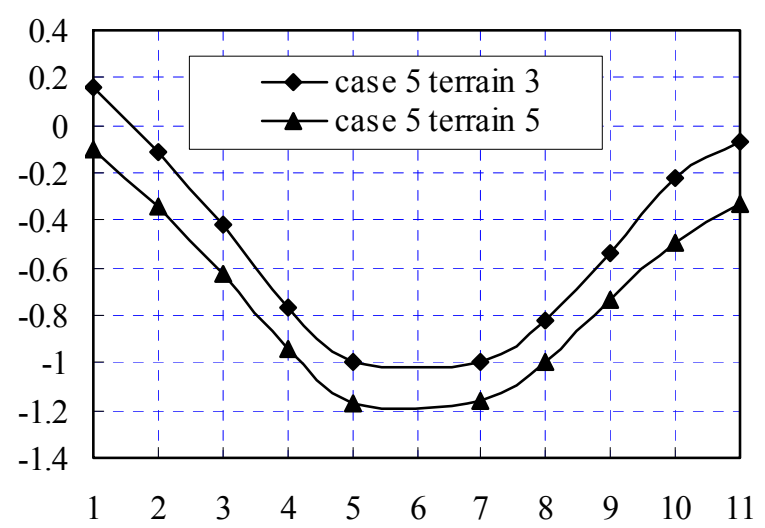

Figure 6. Terrain type effect

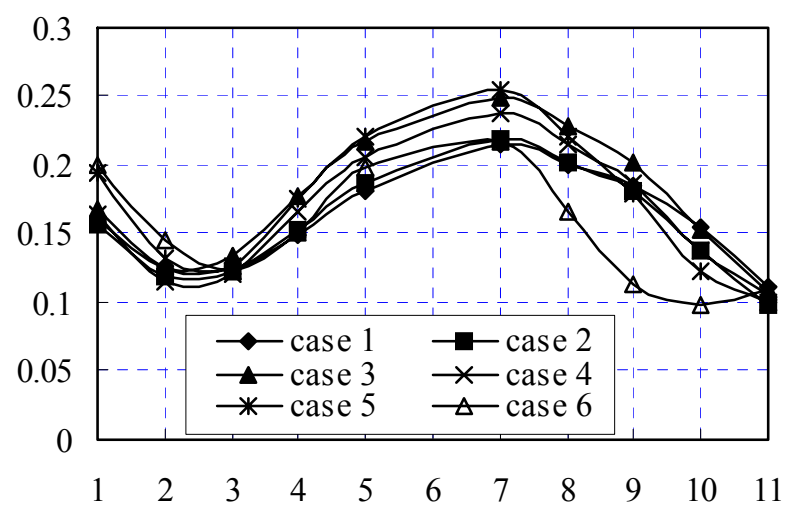

Figure 5. Standard derivation of Model 1, Terrain type Ш

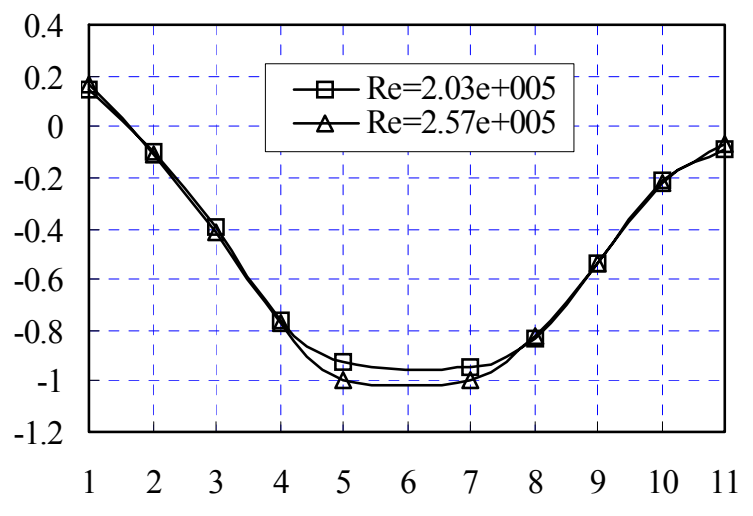

Figure 7. Reynolds number effects (Mean value, Model 1, Case 5, Terrain type Ш)

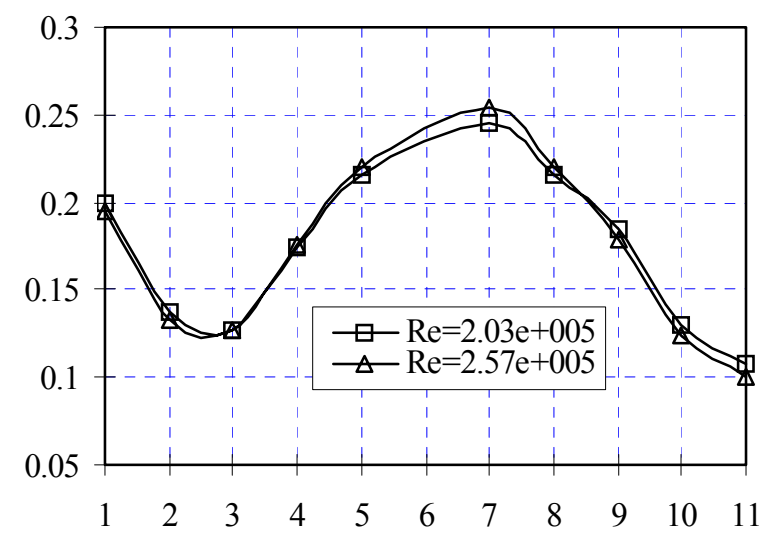

Figure 8. Reynolds number effects (Standard derivation, Model 1 Case 5 Terrain type Ш)

\subsection{Proper Orthogonal Decomposition method in application to reconstruction of wind pressure field for reticulated spherical domes}

There are two interpretations of POD at present. The first regards POD as Karhunen-loeve decomposition (KLD) and the second considers that it consists of three methods: KLD, Principal Component Analysis (PCA) and Singular Value Decomposition (SVD). 


\subsubsection{POD (Karhunen-Loeve decomposition) re-derivation in discrete form}

Assume there are $n$ taps (Figure 9) on the surface of the structure model for wind pressure measurement.

$$
\sum_{i=1}^{n} p\left(x_{i}, y_{i}, z_{i}, t\right) \phi\left(x_{i}, y_{i}, z_{i}\right) \Delta S_{i}=\max
$$

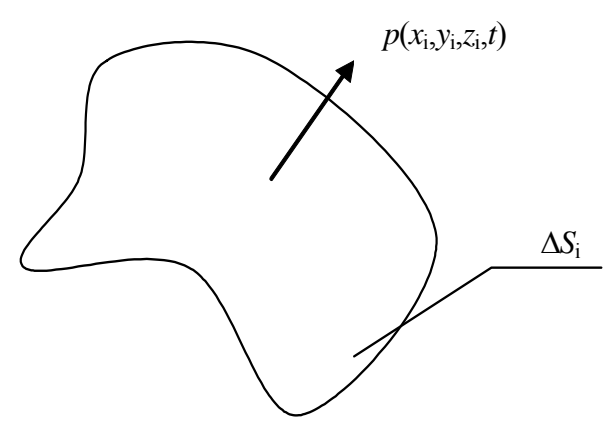

Figure 9. A tap for wind pressure measurement

This equation indicates that the fluctuating wind pressures should be measured synchronously (Condition 1).

$$
\begin{gathered}
\frac{\sum_{i=1}^{n} p\left(x_{i}, y_{i}, z_{i}, t\right) \phi\left(x_{i}, y_{i}, z_{i}\right) \Delta S_{i}}{\left(\sum_{i=1}^{n}\left(\phi\left(x_{i}, y_{i}, z_{i}\right) \Delta S_{i}\right)^{2}\right)^{1 / 2}}=\max \\
\frac{\left(\sum_{i=1}^{n} p\left(x_{i}, y_{i}, z_{i}, t\right) \phi\left(x_{i}, y_{i}, z_{i}\right) \Delta S_{i}\right)^{2}}{\sum_{i=1}^{n}\left(\phi\left(x_{i}, y_{i}, z_{i}\right) \Delta S_{i}\right)^{2}}=\lambda
\end{gathered}
$$

In order to look for the proper function of $\phi(x, y)$, both sides of the above equation are divided by $\delta \phi$.

$$
\frac{\delta}{\delta \phi}\left[\left(\sum_{i=1}^{n} p\left(x_{i}, y_{i}, z_{i}, t\right) \phi\left(x_{i}, y_{i}, z_{i}\right) \Delta S_{i}\right)^{2}-\lambda \sum_{i=1}^{n}\left(\phi\left(x_{i}, y_{i}, z_{i}\right) \Delta S_{i}\right)^{2}\right]=0
$$

- If $\Delta S_{i}=\Delta S_{j}(i, j=1,2, \cdots, n, i \neq j)$ is satisfied first, $\Delta S_{i}$ can be removed from the above equation. Thus, this condition requires the distribution of taps to be uniform (Condition 2).

If the fluctuating component of wind is considered as a steady random process with zero mean value (Condition 3), Eq. (4) is simplified as follows.

$$
\left\lfloor R_{p}\{\phi \phi\}=\lambda\{\phi\}\right.
$$

In Eq. (5) the spatial correlation matrix $\left[R_{p}\right]$ is symmetric.

Eq. (5) is a familiar form of eigenvalue problem. There are several developed algorithms to get the eigenpairs of matrix $\left[R_{p}\right]$ such as the subspace iteration method, the Lancosos method, the inverse iteration method, and so forth.

- If $\Delta S_{i}=\Delta S_{j}(i, j=1,2, \cdots, n, i \neq j)$ is not always satisfied, $\Delta S_{i}$ can not be removed from Eq. (4). 
If the fluctuating component of wind is considered as a steady random process with zero mean value (Condition 3), Eq. (4) is simplified as follows.

$$
\left\lfloor R_{p}\right\rfloor\left\{\phi^{\prime}\right\}=\lambda\left\{\phi^{\prime}\right\}
$$

where $\left\{\phi^{\prime}\right\}=\left\{\begin{array}{c}\phi\left(x_{1}, y_{1}, z_{1}\right) \Delta S_{1} \\ \phi\left(x_{2}, y_{2}, z_{2}\right) \Delta S_{2} \\ \vdots \\ \phi\left(x_{n}, y_{n}, z_{n}\right) \Delta S_{n}\end{array}\right\}$

\subsubsection{Physical meaning of $\left\{\phi^{\prime}\right\}$ in Eq. (6) and discussion when taps non-uniformly distributed}

In terms of POD, the complex time and spatially varying wind pressure field on a building can be divided into time-varying parts $a_{j}(t)$ independent of spatial position and spatially varying 'modes' $\left\{\phi_{j}^{\prime}\right\}$ that depend on spatial position, $j$, and the tributary area, i.e., the geometry of the model surface, but independent of time.

It is noted that in Eq. (7) the eigenvector is weighted by the tributary area of the taps. This is very different from the two methods proposed by Jeong et al. [11]. Modification of the covariance matrix in reference [11] will give rise to a different physical meaning of the principal coordinate/eigenvector and a different optimal problem. In section 2 of reference [11], the weighted-POD of taps non-uniformly distributed of the wind pressure field is denoted by Eq. (8).

$$
\sum_{i=1}^{n} R_{p}^{t}\left(x_{j}, x_{i}\right) \phi^{t}\left(x_{i}\right)=\lambda \phi^{t}\left(x_{j}\right)
$$

where $R_{p}^{t}\left(x_{j}, x_{i}\right)=W\left(x_{j}\right)^{1 / 2} R_{p}\left(x_{j}, x_{i}\right) W\left(x_{i}\right)^{1 / 2}, \phi^{t}\left(x_{i}\right)=W\left(x_{i}\right) \phi\left(x_{i}\right)$ are the modified correlation matrix and eigenvector. Eq. (8) can be rewritten as Eq. (9).

$$
\left[R_{p}\right]\left\{\phi^{\prime}\right\}=\lambda\{\phi\}
$$

while Eq. (9) can be derived from Eq. (10).

$$
\frac{\sum_{i=1}^{n} p\left(x_{i}, y_{i}, z_{i}, t\right) \phi\left(x_{i}, y_{i}, z_{i}\right) \Delta S_{i}}{\left(\sum_{i=1}^{n}\left(\phi\left(x_{i}, y_{i}, z_{i}\right)\right)^{2}\right)^{1 / 2}}=\max
$$

When comparing Eq. (10) with Eq. (1), it is found that they are obviously two mechanical problems. Eq. (10) describes the wind force field on the building surface other than the wind pressure field as depicted in Eq. (1). In terms of the physical meaning of mode $\left\{\phi^{\prime}\right\}$, as mentioned above, in our opinion, it is not rational to regard Eq. (10) as a wind pressure field by proper orthogonal decomposition.

A comprehensive introduction of reconstruction of wind pressure field with consideration of the non-uniform tributary area is presented as follows. 


\subsubsection{Reconstruction of wind pressure field with respect to non-uniformly distributed taps}

Assume that there are $n$ taps non-uniformly distributed on the surface of the test model and $k$ is the sampling time. We can reconstruct the wind pressure field of the test model in terms of POD, Eq. (11), where $p\left(x_{i}, y_{i}, z_{i}, t\right)$ is the wind pressure time history of the test model, $\phi_{i}^{\prime}$ is the eigenvector of covariance matrix, i.e., mode, and $a_{i}(t)$ is the principal coordinate.

$$
\left\{\begin{array}{c}
p\left(x_{1}, y_{1}, z_{1}, t\right) \\
p\left(x_{2}, y_{2}, z_{2}, t\right) \\
\vdots \\
p\left(x_{n}, y_{n}, z_{n}, t\right)
\end{array}\right\}_{n \times k}=\left\{\begin{array}{llll}
\phi_{1}^{\prime} & \phi_{2}^{\prime} & \cdots & \phi_{n}^{\prime}
\end{array}\right\}_{n \times n}\left\{\begin{array}{c}
a_{1}(t) \\
a_{2}(t) \\
\vdots \\
a_{n}(t)
\end{array}\right\}_{n \times k}
$$

If the values of $\phi_{i}^{\prime}$ at the nodes of the dome's network are interpolated or extrapolated from those at the tap locations, we get Eq. (12) as follows.

$$
\left\{\begin{array}{c}
p i\left(x_{1}, y_{1}, z_{1}, t\right) \\
\operatorname{pi}\left(x_{2}, y_{2}, z_{2}, t\right) \\
\vdots \\
\operatorname{pi}\left(x_{l}, y_{l}, z_{l}, t\right)
\end{array}\right\}_{l \times k}=\left\{\begin{array}{llll}
\phi_{1}^{\prime i} & \phi_{2}^{\prime i} & \cdots & \phi_{n}^{\prime i}
\end{array}\right\}_{l \times n}\left\{\begin{array}{c}
a_{1}(t) \\
a_{2}(t) \\
\vdots \\
a_{n}(t)
\end{array}\right\}_{n \times k}
$$

where $\operatorname{pi}\left(x_{i}, y_{i}, z_{i}, t\right)$ is the simulated wind pressure time history for the practical structure, $\phi_{j}^{\prime i}$ is the interpolated eigenvector of the covariance matrix, and $l$ is the node number of a practical dome structure in reduced scale. $l$ can be larger or smaller than $n$. It is noted here that the taps are non-uniformly distributed. $\phi_{i}^{\prime}$ should not be divided by the tributary area before interpolation. This may be questioned. It seems that there would be no difference between uniformly and non-uniformly distributed taps. In the authors' opinion, the geometry information (tributary area) is inherent in the eigenvector matrix $\left\{\phi_{i}^{\prime}\right\}$, and the interpolation is within the column subspace of $\left\{\phi_{i}^{\prime}\right\}$. It would be wrong to remove the tributary area from $\phi_{i}^{\prime}$ before interpolation of POD modes. The steps are as follows. Actually, in this paper the wind pressure time history has been transformed to wind pressure coefficient time history including the mean one since the pressure coefficients are non-dimensional.

Step 1. Input the nodal coordinates and tributary area on the surface of a practical structure in reduced scale (reticulated shell, cable dome, and suspen-dome) and test model;

Step 2. Input the original wind pressure time history on the taps of the spherical dome model measured in ABLWT (different exposure type, height of surrounding wall, reference wind speed). Calculate the covariance of wind pressure time history (exclude mean value, with tributary area). POD does not include the mean value component of the wind pressure time history. It is indispensable to interpolate the mean value for the reduced scale practical structure mesh shape and distribution;

Step 3. POD analysis of wind pressure data on the test model Eq. (6);

Step 4. Scattered data interpolation of eigenvector to $l$ dimension in terms of the nodal coordinates of a practical structure (spatial location of nodes). The cubic interpolation method is adopted in this paper and the linear interpolation method is verified to be worse than the cubic one;

Step 5. Reconstruction/simulation of wind pressure field on the surface of a practical structure in reduced scale;

Step 6. Output the data file of wind pressure history on the surface of the practical structure in reduced scale. 


\subsubsection{Validation}

The spherical dome ABLWT wind pressure test data of half of the taps (K3-6, Figure 10) were utilized to reconstruct the wind pressure field, and they are compared with those for all the taps (K6-6, Figure 3) for validation of Eq.(1) Eq.(7). K3-6 includes half of the taps of K6-6. The first mode is predominant. Figure 11 gives the time history of reconstruction by using and the corresponding measured one at node 3 (K6-6).

Before interpolation, $\phi_{i}^{\prime}$ should not be divided by the tributary area. The geometry information (tributary area) is inherent in the eigenvector. Moreover, the interpolation is within the column space of the

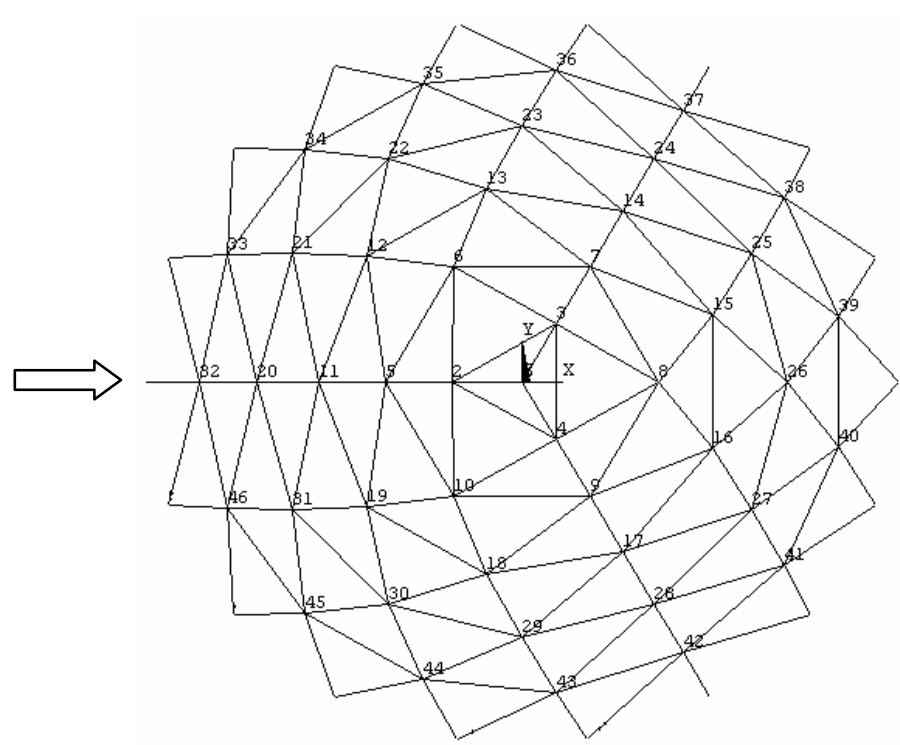

Figure 10. Node numbering (K3-6) eigenvector matrix. The error in the reconstructed time history of the wind pressure coefficients is due to the error of scattered data interpolation of the POD modes. Linear interpolation causes much bigger errors than cubic interpolation. The error of POD mode interpolation increases as the corresponding eigenvalue decreases. The real POD modes are smoother than the interpolated ones. It is considered in reference [12] that the precision of the reconstruction of wind pressure time history on the surface of practical structures depends on not only scattered data interpolation methods but also on the density of taps on a test model.

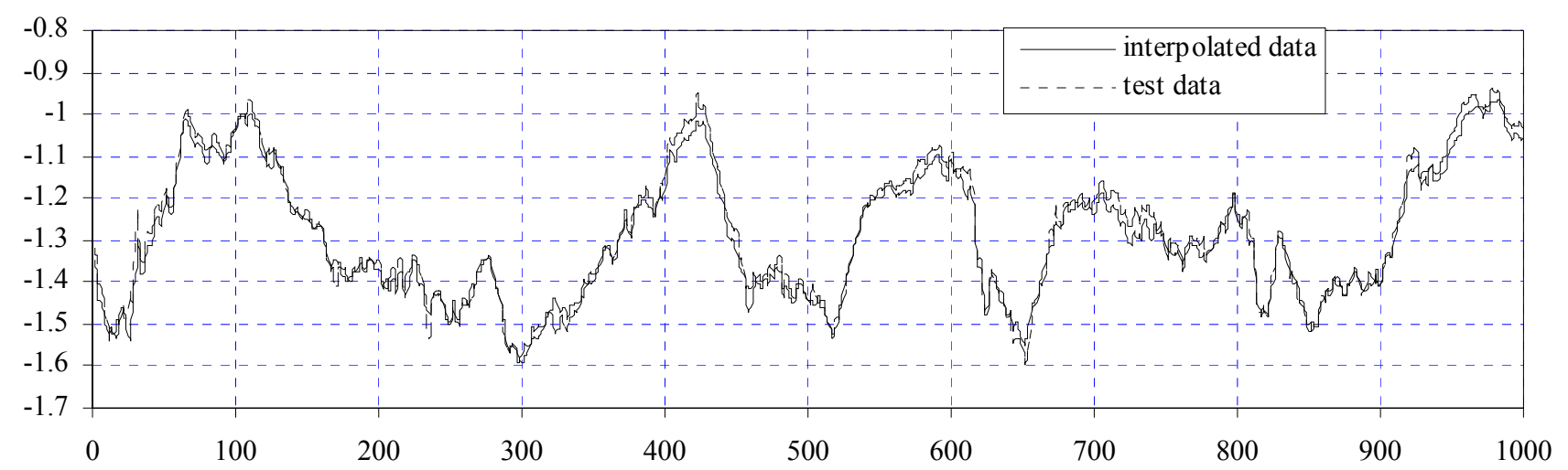

Figure 11. Time history of wind pressure coefficient at node 3 (1s, interpolated from K3-6 to K6-6 based on POD)

\subsection{Wind-induced vibration analysis of single-layer reticulated shells using mode superposition}

The second objective of the wind tunnel test was to study wind effects on practical structures.

\subsubsection{Motion equation and mode superposition method}

The dynamic motion equation can be written as follows. 


$$
[M]\{\ddot{Y}(t)\}+[C]\{\dot{Y}(t)\}+[K]\{Y(t)\}=\left[\phi^{\prime}\right]\{a(t)\}=\{P(t)\}
$$

where $[M]$ is the mass matrix, $[C]$ is the damping matrix, $[K]$ is the stiffness matrix, and $\{Y(t)\}$ is the displacement response of the structure. [ $\left.\phi^{\prime}\right]$ is the POD mode matrix, $\{a(t)\}$ is a matrix of principle coordinates. All possible types of time-dependent load, for example seismic, sea wave and wind, can be represented by the sum of orthogonal load modes that are independent of time. $\{P(t)\}$ is the external fluctuating load.

Assume a displacement solution in the form of Eq. (14)

$$
\{Y(t)\}=[\varphi]\{q(t)\}
$$

where $[\varphi]$ is designated as a modal matrix, which is also independent of time, and $\{q(t)\}$ denotes the modal coordinates. Substitute Eq. (14) into Eq. (13) and pre-multiply by $[\varphi]^{T}$, thus

$$
[\varphi]^{T}[M][\varphi]\{\ddot{q}(t)\}+[\varphi]^{T}[C][\varphi]\{\dot{q}(t)\}+[\varphi]^{T}[K][\varphi]\{q(t)\}=[\varphi]^{T}\left[\phi^{\prime}\right]\{a(t)\}
$$

where $[m p f]=[\varphi]^{T}\left[\phi^{\prime}\right]$ is termed modal participation factor matrix.

\subsubsection{New mode-load-correlation matrix on the basis of POD modes and eigenvalues}

For wind loading, it is indispensable to determine how many vibration modes are adequate to be included in mode superposition transient analysis. This gives rise to many kinds of modal truncation methods. This section gives out a new matrix to detect the spatial distribution of the structural response. The static/dynamic load participation factors proposed by Wilson [23] are very interesting. However, they need to make quasi-static/dynamic assumption of unknown variables.

\section{(1) Square of external work associated with fluctuating wind loading}

In order to determine the predominant mode, in reference [19] the authors checked the correlation coefficient between the dominant modes and the dominant frequency of the principal coordinates obtained by Proper Orthogonal Decomposition (POD) of the fluctuating pressure fields acting on the dome model and the mode shapes and the natural frequencies of the dome structures obtained by Finite Element Method (FEM) analysis. It is verified that it is necessary to take into account the effect of the principal coordinates $\{a(t)\}$ in Eq. (11). A clear and interesting derivation is presented as follows.

The total external work associated with wind load at time $t$ is expressed in Eq. (16). The square of the total external work can be rewritten in the form shown in Eq. (17).

$$
\begin{aligned}
& E_{L}(t)=\frac{1}{2}\{Y(t)\}^{T}\{P(t)\}=\frac{1}{2}\{P(t)\}^{T}\{Y(t)\} \\
& E_{L}^{2}(t)=\frac{1}{2}\{Y(t)\}^{T}\{P(t)\} \times \frac{1}{2}\{P(t)\}^{T}\{Y(t)\}=\frac{1}{4}\{Y(t)\}^{T}\left(\{P(t)\}\{P(t)\}^{T}\right)\{Y(t)\}
\end{aligned}
$$

Substitute Eq. (12) and Eq. (1) to Eq. (15), then

$$
E_{L}^{2}(t)=\frac{1}{4}\{q(t)\}^{T}[\varphi]^{T}\left(\left[\phi^{\prime}\right]\{a(t)\}\{a(t)\}^{T}\left[\phi^{\prime}\right]^{T}\right)[\varphi]\{q(t)\}
$$

On the basis of POD analysis [24]

$$
E\left(\{a(t)\}\{a(t)\}^{T}\right)=[\operatorname{Diag}(\lambda)]
$$

where $E($ ) denotes the mathematic expect in statistics. Substitute [mpf] into Eq. (18)

$$
E_{L}^{2}(t)=\frac{1}{4}\{q(t)\}_{1 \times k}^{T}[m p f]_{k \times m}\left(\{a(t)\}\{a(t)\}^{T}\right)_{m \times m}[m p f]_{m \times k}^{T}\{q(t)\}_{k \times 1}, \quad(0<k \leq n)
$$

where $k$ is the number of modes truncated for approximation of structural response, and $n$ is the 
number of degrees of freedom of the whole structural system. On the right side of Eq. (20), only the modal coordinate $\{q(t)\}$ is unknown. Thus, if we eliminate the modal coordinate items and average the time history, a new matrix is given out, as Eq. (21).

$$
[m p f]_{k \times m} E\left(\{a(t)\}\{a(t)\}^{T}\right)_{m \times m}[m p f]_{m \times k}^{T}, \quad(0<k \leq n)
$$

Substitute Eq. (19) into Eq. (21), and Eq. (21) can be simplified as follows Eq. (22).

$$
[m p f]_{k \times m}[\operatorname{Diag}(\lambda)]_{m \times m}[m p f]_{m \times k}^{T}, \quad(0<k \leq n)
$$

Thus, a new indicator for the predominant vibration mode effect can be defined as

$$
[M L C]=[\varphi]_{k \times n}^{T}\left[\phi^{\prime}\right]_{n \times m}[\operatorname{Diag}(\sqrt{\lambda})]_{m \times m}, \quad(0<k \leq n)
$$

where $[M L C]$ is designated by the authors as mode-load-correlation matrix. Then

$$
[m p f]_{k \times m} E\left(\{a(t)\}\{a(t)\}^{T}\right)_{m \times m}[m p f]_{m \times k}^{T}=[M L C][M L C]^{T}, \quad(0<k \leq n)
$$

\section{(2) Physical meaning of mode-load-correlation matrix}

It is noted here that POD is utilized in Eq. (18). By virtue of the physical meaning of POD analysis, the mean value should be excluded in $\{F(t)\}$. Thus, in Eq. (20) the modal coordinate vector $\{q(t)\}$ includes only two parts of structural response with respect to wind-induced vibration, for example, the background response $\{q(t)\}_{B}$ and the resonant response $\{q(t)\}_{R}$. The mean wind load effect $\{q(t)\}_{\text {mean }}$ is not included. It is not difficult to follow that $\{q(t)\}$ is weighted by $[M L C]$. Thus, we conclude that [MLC] is the spatial distribution of the fluctuating structural response that is independent of time. The advantage of this matrix is that it only takes into account of the known variables in the motion equation, without any quasi-static or dynamic assumption.

\section{(3) Numerical example}

In order to verify this point, a numerical example of a single layer reticulated shell (Kiewitt-type, K6-6) with span $120 \mathrm{~m}$, rise $24 \mathrm{~m}$ and lattice type as shown in Figure 2. The section of all elements of the shell (steel tube) is $\Phi 200 \times 8 \mathrm{~mm}$. The density of the material is $7860 \mathrm{Kg} / \mathrm{m}^{3}$. The elastic modulus is $2.1 \times 10^{5} \mathrm{MPa}$.

Time history analysis using the Newmark- $\beta$ method is carried out first. Second, referenced with the results from time history analysis, modal superposition transient analysis is conducted again. The strain energy of each mode accumulated along the time history and kinetic energy are obtained. Figure 12 shows the 91st and 93rd modes of a K6-6 reticulated spherical shell (rise-to-span ratio $1 / 5$, span $30 \mathrm{~m}$ ). The $91 \mathrm{st}$ mode is integrally rising up (see [14]). It should be pointed out that this mode is usually speculated as the 'fundamental mode' of the whole structure. However, the order of this mode depends upon the structure type, rise-to-span ratio, boundary conditions and so forth. The 92nd mode is integrally a torsional mode and the 93rd mode is an anti-symmetric mode of the whole structure. Figure 13 shows the natural frequency. Figures 14 and 15 show the strain energy and kinetic energy distribution of each vibration mode. The peak value is at the 91 st order, which is very interesting. It seems that this special mode contributes most to the background response and significantly to the resonant part. 

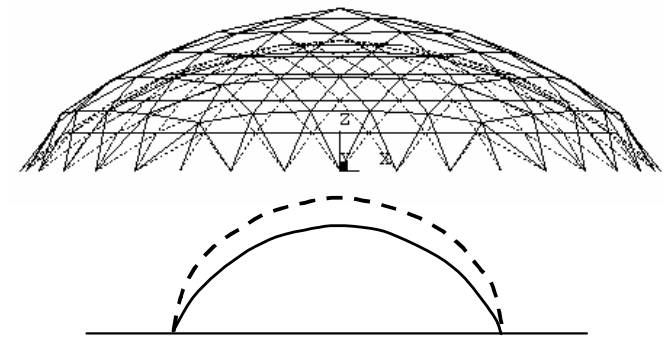

$91^{\text {st }}$ mode
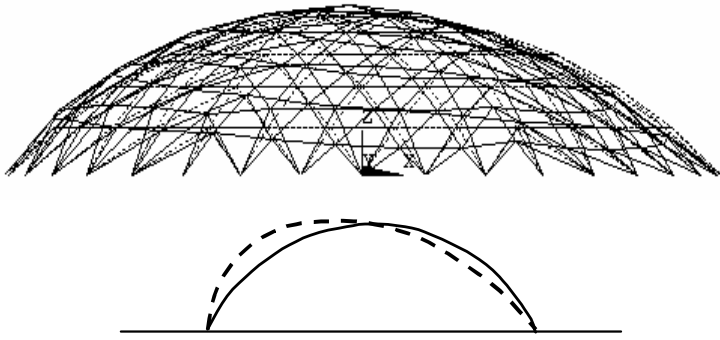

$93^{\text {rd }}$ mode

Figure 12. $91^{\text {st }}$ and $93^{\text {rd }}$ vibration mode of K6-6

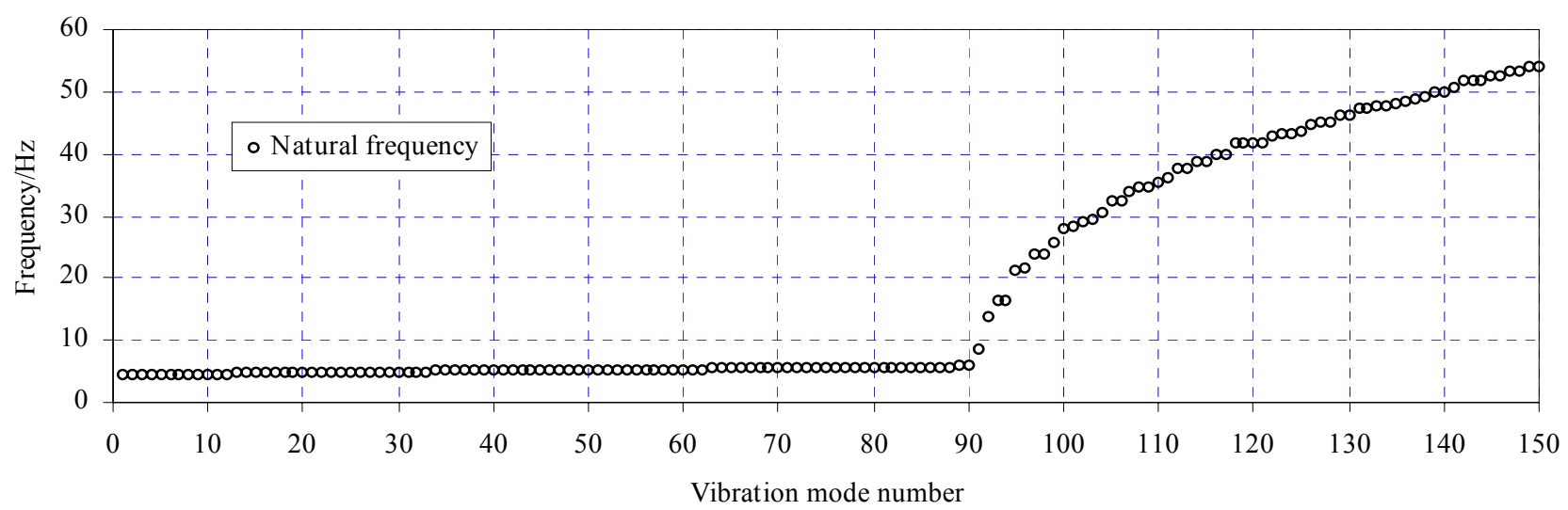

Figure 13. Natural frequency of K6-6

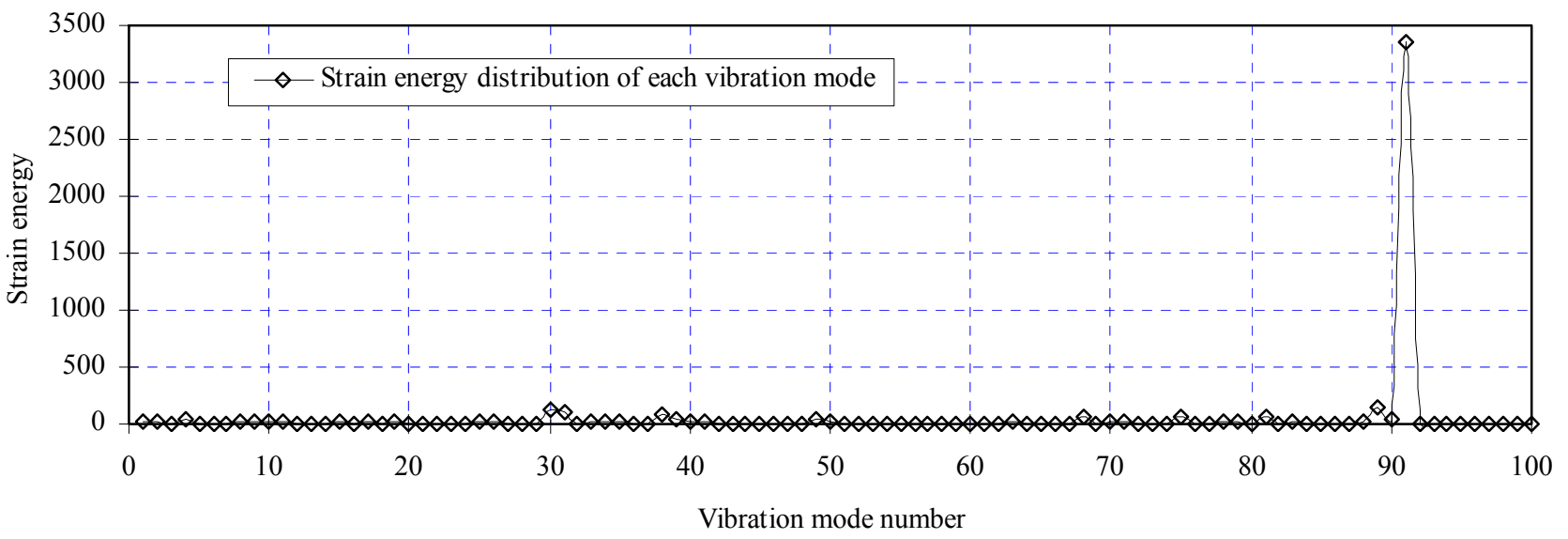

Figure 14. Strain energy distribution of each mode

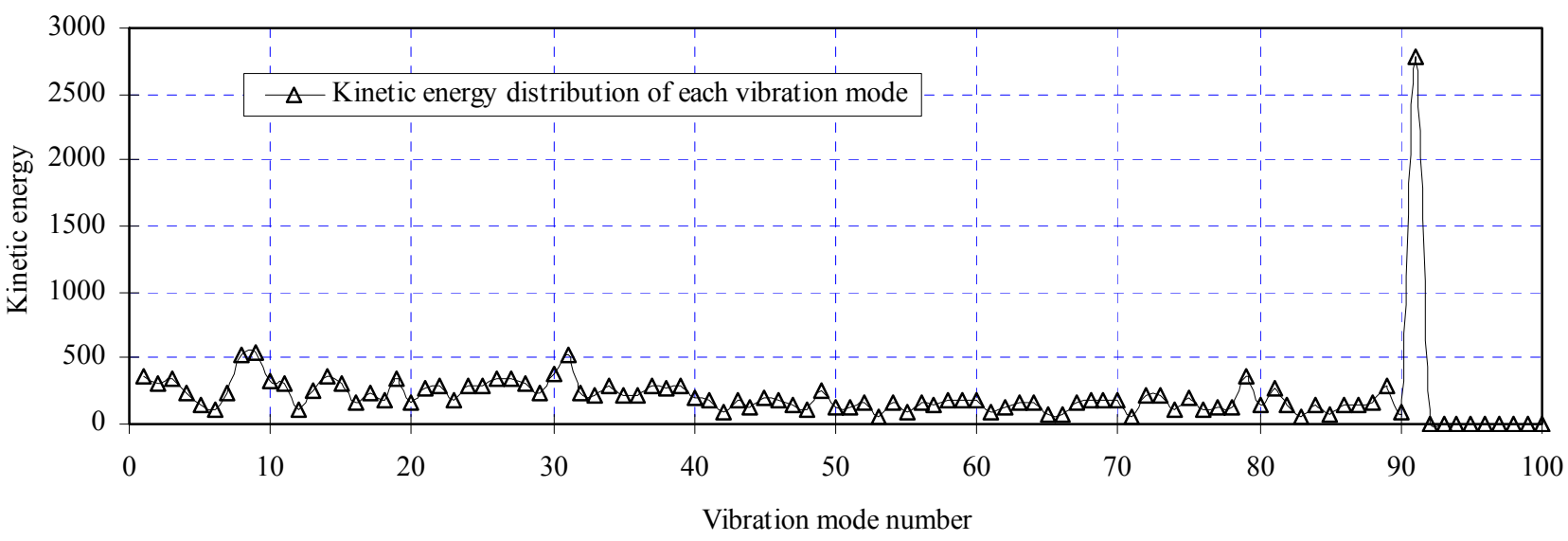

Figure 15. Kinetic energy distribution of each mode 
Figure 16 shows the results of the correlation coefficient of vibration mode (1 100) and POD mode vector (1 91) weighted by eigenvalues from POD analysis. In Figure 16, the 91st POD mode corresponds to the largest eigenvalue. The peak value is at the 91st vibration mode. This is consistent with Figures 14 and 15. This is a new way to find the predominant mode before modal superposition transient analysis. It also suggests that the POD mode with the largest eigenvalue can be utilized to find out a Ritz vector base other than the mean wind load in reference [15].

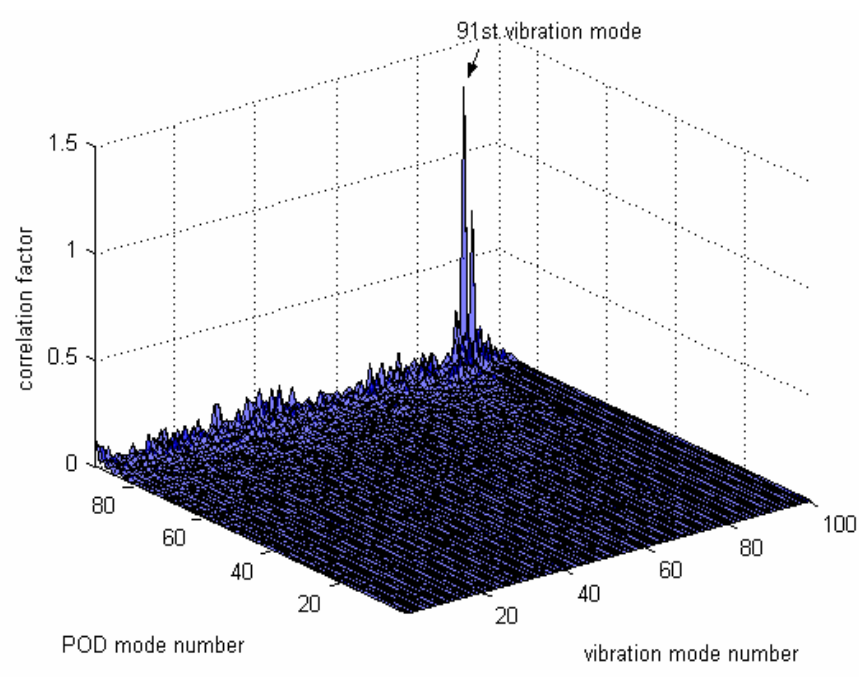

Figure 16. Mode-Load-Correlation

The authors are also very interested in the reason for high mode effects on wind-induced vibration analysis of single-layer reticulated shells. A pseudo-continuum thin shell model is checked for the vibration mode shape and natural frequency distribution of single-layer reticulated spherical shells. It gives out the reason why a mode contributing most to the quasi-static response and significantly to the resonant part ranks as a very high order. Actually, it is because of the membrane-like characteristics of the single-layer reticulated shells. The order of this mode is usually around the total number of unconstrained nodes for low rise-to-span ratio cases. The mode shape is wholly rising up. Further details will be presented in another paper in the near future.

\subsection{3. $[\mathrm{MLC}]$ in application to background response}

For structures in which resonant response is neither significant nor dominant (and can thus be neglected) Holmes [25] applied POD into computation of the covariance of background response. Section 4.2.2 is devoted to finding an effective indicator [MLC] for mode truncation. In this section [MLC] in application to background response is discussed. Regarding background response, the mechanical admittance is consider to be unity. The motion equation can be re-written as in Eq. (25).

$$
\begin{gathered}
\left\{\begin{array}{r}
{\left[\operatorname{Diag}\left(\omega^{2}\right)\right]\{q(t)\}=\{F(t)\}=[\varphi]^{T}\{P(t)} \\
\{\ddot{q}(t)\}+[\operatorname{Diag}(2 \xi \omega)]\{\dot{q}(t)\}=0
\end{array}\right. \\
\{q(t)\}=\left[\operatorname{Diag}\left(\omega^{2}\right)\right]^{-1}[\varphi]^{T}\{P(t)\} \\
E\left(\{q(t)\}\{q(t)\}^{T}\right)=E\left(\left[\operatorname{Diag}\left(\omega^{2}\right)\right]^{-1}[\varphi]^{T}\{P(t)\}\{P(t)\}^{T}[\varphi]\left[\operatorname{Diag}\left(\omega^{2}\right)\right]^{-1}\right)
\end{gathered}
$$

Substitute Eq. (24) into Eq. (28), then

$$
E\left(\{q(t)\}\{q(t)\}^{T}\right)=\left[\operatorname{Diag}\left(\omega^{2}\right)\right]^{-1}[M L C][M L C]^{T}\left[\operatorname{Diag}\left(\omega^{2}\right)\right]^{-1}=\left[R_{q_{i} q_{j}}(0)\right]
$$

Thus

$$
E\left(\{Y(t)\}\{Y(t)\}^{T}\right)=[\varphi] E\left(\{q(t)\}\{q(t)\}^{T}\right)[\varphi]^{T}=[\varphi]\left[R_{q_{i} q_{j}}(0)\right][\varphi]^{T}
$$

A simple and concise formula for computation of background response is shown in Eq. (30) in the time domain. For large-span space structures in which resonant response is either significant or dominant and the corresponding background response is not negligible, basically, no simplified method is convincing at present except transient time history analysis. Further work is needed in future. 


\section{CONCLUSIONS}

Wind pressure distributions on spherical roofs including mean and standard deviation have been presented. Effects on wind pressure distribution of rise- or wall-height-to-span ratio, terrain type and Reynolds number have been investigated. With decrease in wall height, the curves integrally move upward since the turbulence intensity increases while the mean wind velocity decreases. The effect on the pressure distribution of different terrain types is significant. Regarding Reynolds number effect, it is proposed to carefully select proper reference wind velocities for ABLWT tests on streamlined structures.

The second objective of this paper was to investigate wind effects on large-span reticulated spherical shells by a mode superposition method. A new way to determine the special mode that contributes most to wind effects has been put forward. This mode seems to contribute most to background response and significantly to the resonant part. A new matrix (mode-load-correlation matrix) has been proposed. It is concluded that this matrix reveals the spatial distribution of fluctuating structural response including the background response and the resonant response. A simple derivation with respect to mode-load-correlation matrix and background response has been presented.

\section{ACKNOWLEDGEMENTS}

This study was funded by the Ministry of Education, Culture, Sports, Science and Technology, Japan, through the "Academic Frontier" Project, 2000-2004.

\section{REFERENCES}

[1] Seiichi Taniguchi, Hiroshi Sakamoto et al., "Time-averaged aerodynamic acting on a hemisphere immersed in a turbulent flow", International journal of wind engineering and industrial aerodynamics, 1982, 9, pp.257-273.

[2] Toy, N., Moss, W.D. and Savory, E., "Wind tunnel studies on a dome in turbulent boundary layers", International journal of wind engineering and industrial aerodynamics, 1983, 11, pp.201-212.

[3] Toy, N. and Tahouri, B., "Pressure distributions on semi-cylindrical structures of different geometrical cross-sections", International journal of wind engineering and industrial aerodynamics, 1988, 29, pp.263-272.

[4] Newman, B.G. and Ganguli, U. "Flow over spherical inflated buildings", International journal of wind engineering and industrial aerodynamics, 1984, 17, pp.305-327.

[5] Cook, N.J., "The designer's guide to wind loading of building structures. Part 1: Background, damage survey, wind data and structural classification”, Building research establishment report, 1985.

[6] Taylor, T.J., "Wind pressures on a hemispherical dome", International journal of wind engineering and industrial aerodynamics, 1991, 40, pp.199-213.

[7] Hongo, T., "Experimental study of wind forces on spherical roofs", Ph.D. thesis, Tohoku University, 1995. (In Japanese)

[8] Yasushi Uematsu, Motohiko Yamada, Akira Inoue, Takeshi Hongo, "Wind loads and wind-induced dynamic behavior of a single-layer latticed dome", International journal of wind engineering and industrial aerodynamics, 1997, 66, pp.227-248. 
[9] Yasushi Uematsu, Osamu Kuribara, Motohiko Yamada, Akihiro Sasaki, Takeshi Hongo, "Wind induced dynamic behavior and its load estimation of a single-layer latticed dome with a long span", International journal of wind engineering and industrial aerodynamics, 2001, 89, pp.1671-1687.

[10] Carassale, L., Hibi, K., Pagnini, L.C., Solari, G., Tamura, Y., "POD analysis of the dynamic wind pressure on a tall building", BBAA V., 2004.

[11] Seung-Hwan Jeong, B. Bienkiewicz and Hee-Jung Ham, "Proper orthogonal decompostion of building wind pressure specified at non uniformly distributed pressure taps", Journal of wind engineering and industrial aerodynamics, 2000, 87, pp.1-14.

[12] Zhang Zhi-hong and Yukio Tamura, "Some problems of proper orthogonal decomposition method in application to reconstruction of wind pressure field for reticulated spherical domes", Proceedings of 10th ACWE, the tenth Americas conference on wind engineering, 31 May 4 June, 2005. Baton rouge, Louisiana, U. S. A. submitted.

[13] Masuda, K., Nakayama, M., et al., "An efficient evaluation of wind response of dome roof through modal analysis method", Proceeding of $13^{\text {th }}$ national symp. on wind engineering, Tokyo, 1994, pp.209-214. (In Japanese)

[14] Masanao Nakayama, Yasuhito Sasaki, Keiji Masuda, Toshiyuki Ogawa, "An efficient method for selection of vibration modes contributory to wind response on dome-like roofs", International journal of wind engineering and industrial aerodynamics, 1998, 73, pp.31-43.

[15] Ni, Z.H., Huang, M.K. and Xie, Z.N., "Wind-induced response of dome-like roof", Proceedings of the third international conference on Advances in Structural Engineering and Mechanics, Seoul, Korea, Sept. 2-4, 2004, pp.763-771.

[16] Wilson, E.L., "A new method of dynamic analysis for linear and nonlinear systems", Finite elements in analysis and design, 1985, 1, pp.21-23.

[17] Wilson, E.L., Yuan, M.W. and Dickens, J.M., "Dynamic analysis by direct superposition of Ritz vectors", Earthquake engineering and structural dynamics, 1982, 10, pp.813-821.

[18] He, Y.L. and Dong, S.L., "A new frequency domain method for wind response analysis of spatial lattice structures with mode superposition", International Journal of Space structures, 2002, 17(1), pp.67-76.

[19] Zhang Zhihong, Yukio Tamura, Masahiro Matsui, Yoshida Tomoya, "Wind tunnel tests and wind induced vibration analysis on spherical domes", Proceedings of the fourth international conference on advances in steel structures, ICASS'05, 13-15 June 2005, Shanghai, China.

[20] Li, Y.Q. and Tamura, Y., "Wind-resistant analysis for large-span single-layer reticulated shells", International Journal of Space Structures, 2004, 19(1), pp.47-59.

[21] Li, Y.Q. and Tamura, Y., "Nonlinear dynamic analysis for large-span single-layer reticulated shells subjected to wind loading", International Journal of Wind Structures, 2005, 8(1), pp.35-48.

[22] Buresti, G., "The effects of surface roughness on the flow regime around circular cylinder", Journal of wind engineering and industrial aerodynamics, 1981, 8, pp.105-114.

[23] Wilson, E.L., "Three dimensional static and dynamic analysis of structures - a physical approach with emphasis on earthquake engineering”, Third edition, Computers and structures, Inc. Berkeley, California, U.S.A., 2000.

[24] Tamura Y., Suganuma, S., Kikuchi, H. and Hibi, K., "Proper orthogonal decompostion of random wind pressure field", Journal of fluids and structures, 1999, 13, pp.1069-1095.

[25] Holmes, J.D., "Effective static load distribution in wind engineering", Journal of wind engineering and industrial aerodynamics, 2002, 90, pp.91-99. 